\title{
Lebensqualität nach Brustkrebs: Erfassung, Relevanz und effektive Interventionen
}

\section{Quality of life after breast cancer: Assessment, relevance and effective interventions}

Autoren

Martina Schmidt, Karen Steindorf

\section{(c) (i) $(-)$}

Institut

Abteilung Bewegung, Präventionsforschung und Krebs; Deutsches Krebsforschungszentrum (DKFZ) und Nationales Centrum für Tumorerkrankungen (NCT), Heidelberg [Division of Physical Activity, Prevention and Cancer; German Cancer Research Center (DKFZ) and National Center for Tumor Diseases (NCT), Heidelberg]

\section{Schlüsselwörter}

Fatigue, sexuelle Probleme, Schlafprobleme, Nebenwirkungen, Langzeitfolgen

Key words

breast cancer, quality of life, patient reported outcomes, fatigue, sexual problems

Bibliografie

TumorDiagn u Ther 2021; 42: 62-67

DOI 10.1055/a-1334-2889

ISSN $0722-219 X$

(C) 2020. The Author(s).

This is an open access article published by Thieme under the terms of the Creative Commons Attribution-NonDerivative-NonCommercial License, permitting copying and reproduction so long as the original work is given appropriate credit. Contents may not be used for commecial purposes, or adapted, remixed, transformed or built upon. (https://creativecommons.org/licenses/by-nc-nd/4.0/)

Georg Thieme Verlag KG, Rüdigerstraße 14,

70469 Stuttgart, Germany

Zitierweise für diesen Artikel Senologie 2020; 17: 88-93;

DOI https://doi.org/10.1055/a-1030-9792.

Korrespondenzadresse

Dr. Martina Schmidt

Abteilung Bewegung, Präventionsforschung und

Krebs (C110)

Deutsches Krebsforschungszentrum (DKFZ), Im Neuenheimer Feld 581, 69120 Heidelberg, Deutschland Tel.: ++49/6221/422220

m.schmidt@dkfz.de

\section{ZUSAMMENFASSUNG}

Gesundheitsbezogene Lebensqualität ist für Brustkrebsbetroffene in allen Stadien, sowohl während als auch mittelund langfristig nach Abschluss der Therapie, von großer Relevanz. Bei Zulassungsstudien für neue Behandlungen sowie wissenschaftlichen Studien zum Vergleich verschiedener Therapien gewinnt Lebensqualität als Endpunkt zunehmend an Bedeutung. Neben einer kurzen globalen Einschätzung der Lebensqualität sollten weitere wichtige Patient-Reported Outcomes erhoben werden. Patientinnen mit Brustkrebs berichten häufig über Einschränkungen durch Fatigue, Schlafprobleme, sexuelle bzw. klimakterische Probleme, kognitive Probleme, psychische Probleme und Verlust an körperlicher Leistungsfähigkeit. Die Lebensqualität nach Brustkrebs könnte vermutlich weiter gesteigert werden, wenn Aufklärung, Screening und Behandlung dieser Symptome systematischer Bestandteil der onkologischen Versorgung würden.

\section{ABSTRACT}

Health-related quality of life is of great relevance for breast cancer patients at all stages, both during treatment and in the medium and long term after treatment. Quality of life is becoming increasingly important as an end point in licensing studies for new treatments and in scientific studies comparing different therapies. In addition to a brief global assessment of quality of life, other important patient-reported outcomes should be assessed. Patients with breast cancer often report limitations due to fatigue, sleep problems, sexual and/or menopausal problems, cognitive problems, mental problems and loss of physical performance. Quality of life after breast cancer could probably be further increased if education, screening and treatment of these symptoms were a systematic part of oncological care. 


\section{Einleitung}

Das Überleben nach Brustkrebs hat sich in den letzten Jahren und Jahrzehnten deutlich verbessert. Die durchschnittliche relative 5-Jahres-Überlebensrate von Frauen mit Brustkrebs liegt in Deutschland bei ca. $88 \%$. Daher ist die langfristige Lebensqualität von großer Bedeutung. Auch während der adjuvanten Therapie spielen Lebensqualitätsaspekte eine wichtige Rolle, unter anderem für die Therapie-Compliance oder bereits bei der Entscheidung zwischen verschiedenen onkologischen Therapien. Dabei möchten Betroffene wissen, wie sich andere Patientinnen während und nach der jeweiligen Therapie gefühlt haben. Im fortgeschrittenen Stadium, wenn keine Heilung mehr möglich ist, ist die Verbesserung bzw. der Erhalt der Lebensqualität sogar das primäre Ziel.

Der Begriff „Quality of Life (QOL)“ tauchte erstmals in den 1960 er-Jahren in der medizinischen Literatur auf. Es brauchte jedoch gut 30 Jahre, bis Lebensqualität als relevanter Endpunkt in klinischen Studien anerkannt wurde. In jüngerer Zeit haben von den Patienten berichtete, subjektiv wahrgenommene Gesundheitszustände, sogenannte Patient-Reported Outcomes (PROs), einschließlich der selbstberichteten gesundheitsbezogenen Lebensqualität, in onkologischen Studien stark an Bedeutung gewonnen. Von der amerikanischen Food and Drug Administration (FDA) und der European Medicines Agency (EMA) wird der Einsatz von PROs in Zulassungsstudien zur Erfassung von Symptomen, Beeinträchtigungen und Nebenwirkungen der Therapie zunehmend gefordert [1]. Die Erfassung der Sicht der Patienten ist essenziell bei der Erhebung der Lebensqualität und Symptombelastung, da die Einschätzungen von Ärzten oft nur mäßig mit den Erfahrungen der Patienten korrelieren.

\section{Begriffe und Methodik}

\section{Definition der gesundheitsbezogenen Lebensqualität}

Der Begriff „Lebensqualität“ ist ein multidimensionales Konstrukt aus physischen, psychischen und sozialen Aspekten. Da die subjektiv empfundene Qualität des Lebens auch von Wohlstand, Freiheit, Politik, Bildung, Kultur und Religion abhängen kann, wird oft der Begriff „gesundheitsbezogene Lebensqualität“ (Health-Related Quality of Life, HRQOL) verwendet, wenn der Einfluss von gesundheitsbezogenen Faktoren betrachtet werden soll.

\section{INFOKASTEN}

Gesundheitsbezogene Lebensqualität (HRQOL) wurde von der WHO 1993 [2] definiert als:

„Die Wahrnehmung der eigenen Lebenssituation im Kontext der Kultur- und Wertesysteme, in denen das Individuum lebt, und in Bezug auf seine Ziele, Standards und Anliegen. Es handelt sich um ein weitreichendes Konzept, das in komplexer Weise von der körperlichen Gesundheit, dem psychosozialen Zustand, dem Grad der Unabhängigkeit, den sozialen Beziehungen und ihrem Verhältnis zu den wichtigsten Merkmalen der Umwelt beeinflusst wird.“
HRQOL gehört zu der Gruppe der Patient-Reported Outcomes (PROs). Einfach ausgedrückt, ist ein PRO ein Maß für die Wahrnehmung des Patienten des eigenen Gesundheitszustandes oder der Lebensqualität.

\section{INFOKASTEN}

Patient-Reported Outcome (PRO) wurde von der FDA definiert als:

„Eine Messung, die auf einem Bericht basiert, der direkt vom Patienten (d. h. von der Versuchsperson) über den Status des Gesundheitszustandes eines Patienten ohne Änderung oder Interpretation der Reaktion des Patienten durch einen Kliniker oder eine andere Person kommt.“

\section{Erfassung der gesundheitsbezogenen Lebensqualität}

HRQOL wird mittels validierter Fragebogen erfasst. Dabei wird zwischen (krankheits)spezifischen und generischen (krankheitsübergreifenden) Instrumenten unterschieden ( $\triangleright$ Tab. 1). Die spezifischen Instrumente sind sensitiver in Bezug auf Veränderungen der betrachteten Erkrankung und werden von Patienten meist als relevanter erachtet als generische Instrumente, da sie die eigenen Probleme besser abbilden. Generische Instrumente ermöglichen dafür krankheitsübergreifende Vergleiche und spielen etwa bei gesundheitsökonomischen Evaluationen eine wichtige Rolle. Das am häufigsten in Nordamerika verwendete krebsspezifische Instrument ist der FACT-Fragebogen, in Europa der EORTC QLQC30. Beide haben viele tumor-, symptom- oder behandlungsspezifische Zusatzmodule und sind in vielen Sprachen validiert.

Die HRQOL kann global durch einen Wert auf einer Skala eingeschätzt werden. Der EORTC QLQ-C30-Fragebogen erfasst die globale HRQOL als Mittelwert von zwei subjektiven Selbsteinschätzungen auf einer Skala von 1 (sehr schlecht) bis 7 (ausgezeichnet) anhand der folgenden Fragen: 1) „Wie würden Sie insgesamt Ihren Gesundheitszustand während der letzten Woche einschätzen?“ und 2) „Wie würden Sie insgesamt Ihre Lebensqualität während der letzten Woche einschätzen?“. Neben der globalen Lebensqualitätseinschätzung werden auch unterschiedliche Funktionsbereiche und Symptome erfasst, die einen potenziellen Einfluss auf die HRQOL haben. Der EORTC QLQ-C30 erhebt zudem die physische, emotionale, soziale und kognitive Funktion und die Rollenfunktion (Beeinträchtigungen in der Arbeit oder Freizeit) sowie typische Symptome während oder nach einer Krebstherapie (Müdigkeit/Fatigue, Schlafstörungen, Schmerzen, Atemnot, Übelkeit/Erbrechen, Appetitlosigkeit, Verstopfung, Durchfall) und finanzielle Auswirkungen.

Kürzlich wurde von der EORTC-Gruppe ein neuer Summenscore eingeführt, der die einzelnen Funktionen und Symptome des EORTC QLQ-C30 gewichtet zusammenfasst. Dieser Summenscore zeigte bessere prognostische Werte für das allgemeine Überleben als der globale HRQOL-Score und jeder andere Score des EORTC QLQ-C30 [3].

Brustkrebsspezifische Funktionen und Symptome werden mit dem EORTC QLQ-BR45 Zusatzmodul erhoben, beispielsweise Beschwerden in der Brust bzw. in Arm/Schulter oder sexuelle Aktivität. Das BR45-Modul ist eine Erweiterung des älteren BR23-Moduls. 
- Tab. 1 Häufig verwendete generische und krebsspezifische Lebensqualitätsfragebogen.

\begin{tabular}{|c|c|c|c|}
\hline Typ & \multicolumn{2}{|l|}{ Instrument } & Items \\
\hline \multirow[t]{4}{*}{ Generisch } & SF-36 & Medical Outcomes Study Short-Form 36 & 36 \\
\hline & EQ-5D-3 L & EuroQol Scale (je 3 Antwortmöglichkeiten, alte Version) & 5 \\
\hline & EQ-5D-5 L & EuroQol Scale (je 5 Antwortmöglichkeiten, seit 2009) & 5 \\
\hline & EQ VAS & EuroQol Scale Visual Analogue Scale & 1 \\
\hline \multirow[t]{5}{*}{ Krebsspezifisch } & EORTC QLQ-30 & $\begin{array}{l}\text { European Organisation for Research and Treatment of Cancer Quality of Life - } \\
\text { Core Questionaire }\end{array}$ & 30 \\
\hline & EORTC QLQ-BR23 & Brustkrebsspezifisches Zusatzmodul (alte Version) & 45 \\
\hline & EORTC QLQ-BR45 & Brustkrebsspezifisches Zusatzmodul (seit 2018) & 45 \\
\hline & FACT-G & Functional Assessment of Cancer Treatment - General & 27 \\
\hline & FACT-B & Functional Assessment of Cancer Treatment - Breast & 37 \\
\hline
\end{tabular}

\section{Referenzwerte}

Zur Einordnung der HRQOL bei Krebspatienten sind Vergleiche mit der Allgemeinbevölkerung hilfreich. Für den EORTC QLQ-C30 liegen dazu normative Werte aus einer repräsentativen Stichprobe $(n=4,684)$ aus der deutschen Bevölkerung stratifiziert nach Alter und Geschlecht vor [4]. Kürzlich wurden zudem Referenzwerte zum EORTC QLQ-C30 für Brustkrebspatientinnen im frühen sowie fortgeschrittenen Stadium, stratifiziert nach unterschiedlichen Kriterien (Alter, Region, Performancestatus, Komorbiditäten), publiziert, die Kliniker zur Interpretation von QOL-Werten ihrer Patientinnen heranziehen können [5].

\section{Interpretation und Analyse der Erhebungen}

Von der Initiative Setting International Standards in Analyzing Patient-Reported Outcomes and Quality of Life Endpoints Data (SISAQOL) werden derzeit Standards für die Erhebung, statistische Analyse und das Berichten von PROs erarbeitet (https://event. eortc.org/sisaqol/conferences-and-publications/). Wie bei jeder Messung müssen mögliche Ungenauigkeiten oder systematische Verzerrungen bei der Erhebung von PROs in Betracht gezogen werden. Möglich ist u. a. ein sogenannter Response-Shift.

\section{INFOKASTEN}

\section{Response-Shift}

Response-Shift bezeichnet eine Veränderung des Bewertungshintergrunds für subjektive Erhebungen im Verlauf von schweren Erkrankungen. Dies kann etwa in Form einer Repriorisierung oder Neukonzeptualisierung auftreten: Werte oder Ziele, die eine Probandin für die Lebensqualität für wesentlich hielt, können nach einer Brustkrebserkrankung quantitativ oder qualitativ anders als vor der Erkrankung bewertet werden. Das könnte das Phänomen erklären, dass die Lebensqualität nach überstandener Brustkrebsbehandlung oft über den Werten vor der Diagnose und über den Vergleichswerten aus der altersbezogenen Allgemeinbevölkerung liegt. ResponseShift kann aber auch infolge einer Rekalibrierung auftreten:

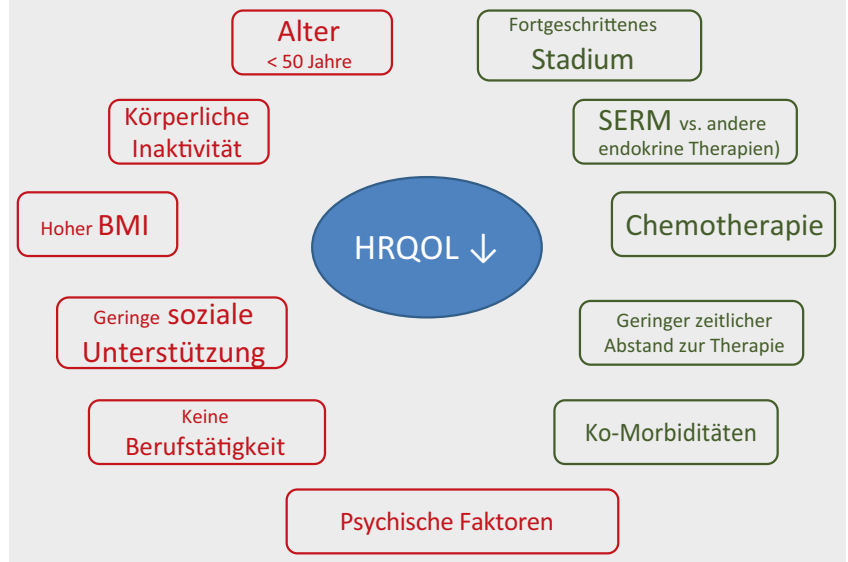

- Abb. 1 Personenbezogene (rot) und klinische (grün) Faktoren, die mit verringerter gesundheitsbezogener Lebensqualität (HRQOL) assoziiert sind.

Patientinnen, die beispielsweise in der akuten Behandlungsphase unter sehr starken Schmerzen gelitten haben, beurteilen später moderate Schmerzen auf einer Skala von 0 bis 10 möglicherweise niedriger, als sie dieselben Schmerzen vor der Akutphase bewertet hätten. Beim Vergleich der Lebensqualität zwischen randomisierten Gruppen spielt der Response-Shift jedoch meist keine wesentliche Rolle.

\section{Epidemiologie}

\section{Determinanten und Kofaktoren der gesundheitsbezogenen Lebensqualität}

Die HRQOL wird allgemein von soziodemografischen Faktoren, und bei Krebspatienten auch von klinischen Faktoren bestimmt ( $\triangleright$ Abb. 1). In der Allgemeinbevölkerung berichten Männer generell bessere HRQOL, höhere Funktionswerte und weniger Symptome als Frauen. Bei beiden Geschlechtern sinken die Funktionswer- 
te und nehmen die Symptome mit steigendem Alter in der Allgemeinbevölkerung zu [4]. Im Gegensatz dazu ist bei Patientinnen mit nicht metastasiertem Brustkrebs niedrigeres Alter mit schlechterer HRQOL bzw. mehr Symptomen assoziiert [6, 7]. Des Weiteren zeigten Studien niedrigere HRQOL bei hohem BodyMass-Index (BMI) und körperlicher Inaktivität [7, 8]. Diese prinzipiell veränderbaren Faktoren sind somit ein nicht unwesentlicher Ansatzpunkt zu Verbesserungen der Lebensqualität. Auch geringe soziale Unterstützung sowie Verlust der Berufstätigkeit sind wichtige Faktoren, die in Bezug auf Lebensqualität im Blick behalten werden sollten. Darüber hinaus gehen psychische Vorbelastungen (frühere oder bestehende depressive Symptome, mentale Erkrankungen) ebenfalls mit stärkeren Verlusten der HRQOL und einer höheren Symptomlast, insbesondere der Fatigue, einher [7, 8]. In einer deutschen Studie bei 2671 Langzeitüberlebenden nach Brustkrebs berichteten $17 \%$ moderate bis große Angst vor einem Rezidiv, was mit negativen Auswirkungen auf die Lebensqualität einherging [9]. Neben relevanten klinischen Aspekten in Bezug auf die Bekämpfung des Tumors sollten daher auch die prädiktiven Faktoren hinsichtlich Lebensqualität nach Brustkrebs betrachtet werden.

Von den üblichen Brustkrebstherapien hat Chemotherapie die stärkste negative Auswirkung auf die HRQOL [6, 8]. Dabei zeigten sich keine starken Unterschiede zwischen Chemotherapien mit oder ohne Taxane [10]. Patientinnen mit Brustkrebs im Frühstadium, die Selektive Estrogen-Rezeptor-Modulatoren (SERMs) erhalten hatten, zeigten mehr depressive Symptome als Patientinnen unter anderen endokrinen Therapien [6]. Der Einfluss der Therapie nimmt jedoch mit der Zeit ab und spielt für die längerfristige Lebensqualität keine große Rolle mehr. Patientinnen im fortgeschrittenen Stadium berichten durchschnittlich eine schlechtere HRQOL als Patientinnen in früheren Stadien [6]. Für die Lebensqualität nach Brustkrebs scheinen Fatigue, Schmerzen, psychische sowie kognitive Probleme besonders relevant zu sein [11, 12].

\section{Verlauf der gesundheitsbedingten Lebensqualität sowie relevanter PROS nach Brustkrebs}

Während der adjuvanten Therapie berichten Brustkrebspatientinnen im Mittel signifikant schlechtere globale Lebensqualität und schlechtere Werte in allen Funktionsbereichen (physische, emotionale, soziale, kognitive und Rollenfunktion), mehr Symptome, insbesondere Fatigue und Schlafstörungen, sowie mehr finanzielle Sorgen als Frauen gleichen Alters in der Allgemeinbevölkerung. Ein oder mehrere Jahre nach Abschluss einer adjuvanten Brustkrebstherapie bewerteten (erkrankungsfreie) Frauen im Mittel ihre Lebensqualität gleich gut oder sogar besser als Frauen vergleichbaren Alters der Allgemeinbevölkerung, jedoch zeigt sich noch eine signifikant höhere Symptomlast [12-14].

- Abb. 2 stellt häufig von Frauen nach Brustkrebs berichtete Probleme dar. Betroffene berichten noch mehrere Jahre nach der Brustkrebsdiagnose beispielsweise über kognitive Beeinträchtigungen wie verminderte Konzentrationsfähigkeit und Gedächtnisprobleme. Die kognitive Funktion war in unterschiedlichen Studien bei Frauen auch mehrere Jahre nach Brustkrebs noch signifikant schlechter als bei Frauen vergleichbaren Alters der Allgemeinbevölkerung [12-15]. Die genauen Ursachen dafür

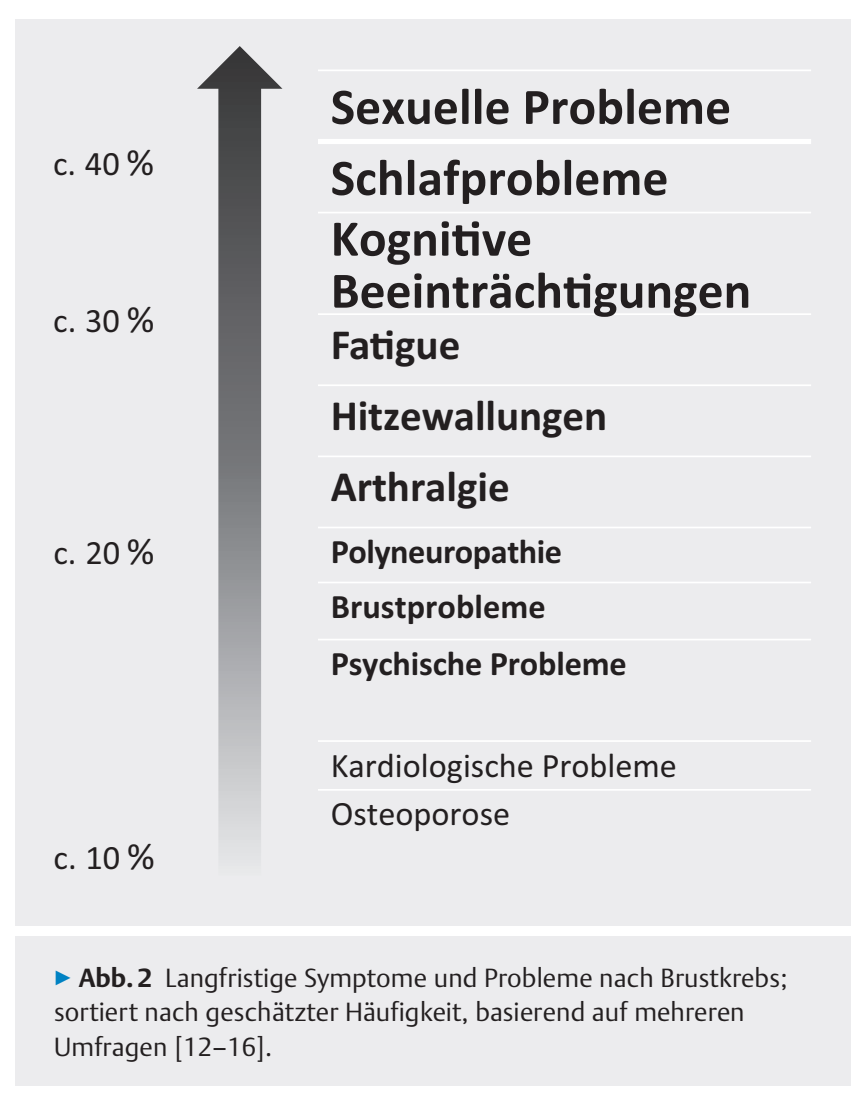

sind noch unklar und werden derzeit weiter erforscht. Darüber hinaus zeigten sich bei erkrankungsfreien Brustkrebsüberlebenden nach 5 Jahren auch signifikant häufiger Schlafprobleme als in der Referenzpopulation [12, 13].

Als besonders häufiges Problem, bei dem sich Frauen nach Brustkrebs mehr Unterstützung wünschen, werden sexuelle Beschwerden (z. B. vaginale Trockenheit, Dyspareunie, Libidoverlust) sowie Hitzewallungen genannt [12, 16]. Klimakterische Beschwerden sind in der Altersgruppe der Betroffenen nicht überraschend. Jedoch zeigte eine Studie bei 843 erkrankungsfreien Frauen rund 6 Jahre nach Brustkrebsdiagnose (ohne endokrine Therapie bzw. bereits abgeschlossen) signifikant schlechtere vasomotorische und sexuelle Scores im Vergleich zu Kontrollen aus der Allgemeinbevölkerung [17].

Eines der häufigsten Probleme während der adjuvanten Therapie ist Fatigue. Prävalenz und Intensität sinken in den Monaten und Jahren nach Therapieende deutlich ab. Jedoch berichtet etwa ein Viertel der erkrankungsfreien Frauen selbst 5 Jahre nach der Brustkrebsdiagnose noch moderate bis starke Fatigue [12, 13]. Die ist ein nicht zu vernachlässigendes Problem, insbesondere weil Fatigue in verschiedenen Untersuchungen die stärkste Beeinträchtigung der HRQOL aufwies und zudem einer der Hauptgründe ist, dass Frauen nach Brustkrebs nicht mehr ins Berufsleben zurückkehren [15]. Prädiktoren für persistierende Fatigue umfassen u. a. mangelnde körperliche Aktivität, starkes Übergewicht, psychische Probleme, Schlafprobleme, Mangel an sozialer Unterstützung, aber auch klimakterische Symptome [8]. Chemotherapie hingegen - einer der wichtigsten Faktoren für das Auftreten von Fatigue - ist kein Prädiktor für ein langfristiges Persistieren der Fatigue. 
- Tab. 2 Nichtmedikamentöse Interventionen mit gut belegten Effekten auf die HRQOL.

\begin{tabular}{|c|c|c|c|}
\hline Intervention & $\begin{array}{l}\text { Gute Evidenz für } \\
\text { Effekte auf: }\end{array}$ & $\begin{array}{l}\text { Wahrscheinliche }{ }^{1} \text { weitere } \\
\text { Effekte auf: }\end{array}$ & Kommentar \\
\hline $\begin{array}{l}\text { Kraft-/Ausdauertraining } \\
\text { [20] }\end{array}$ & $\begin{array}{l}\text { HRQOL, Fatigue, körperliche } \\
\text { Fitness, Angst, Depressivität }\end{array}$ & $\begin{array}{l}\text { Schlaf, Knochendichte, Polyneuropa- } \\
\text { thie, kognitive Funktion, Kardiotoxizität, } \\
\text { Sturzprophylaxe, Schmerzen, sexuelle } \\
\text { Funktion }\end{array}$ & $\begin{array}{l}\text { Trainingsempfehlungen müssen durch } \\
\text { weitere Forschung noch besser } \\
\text { individualisiert werden. }\end{array}$ \\
\hline Yoga [21] & $\begin{array}{l}\text { HRQOL, Fatigue, Schlaf, } \\
\text { Stress, kognitive Funktion }\end{array}$ & $\begin{array}{l}\text { Depression, Angst, Schmerz, } \\
\text { körperliche Fitness, Sturzprophylaxe, } \\
\text { Schmerzen }\end{array}$ & $\begin{array}{l}\text { Welche Yoga-Richtung die besten } \\
\text { Effekte erzielt, muss weiter erforscht } \\
\text { werden. Auch Tai-Chi und Qi Gong } \\
\text { haben z. T. gute Effekte gezeigt. }\end{array}$ \\
\hline $\begin{array}{l}\text { Psychosoziale, edukati- } \\
\text { ve und/oder verhaltens- } \\
\text { basierte Interventionen } \\
\text { (z. B. MBSR, CBT) [19] }\end{array}$ & z. T. für Fatigue, HRQOL & Abhängig von Interventionsausrichtung & $\begin{array}{l}\text { Spezifisch auf bestimmte Symptome } \\
\text { ausgerichtete Interventionen erzielen } \\
\text { oft bessere Effekte für dieses Symptom. }\end{array}$ \\
\hline
\end{tabular}

Die HRQOL kann durch weitere, therapiespezifische Nebenwirkungen eingeschränkt werden wie Arthralgie, eine häufige Nebenwirkung von Aromatasehemmern, oder Polyneuropathie, eine potenzielle Nebenwirkung mancher Chemotherapien. Zudem haben Frauen selbst mehrere Jahre nach einer überstandenen Brustkrebserkrankung häufig eine reduzierte kardiorespiratorische Fitness.

\section{Maßnahmen zur Verbesserung der Lebensqualität nach Brustkrebs}

Die Entwicklung von nebenwirkungsärmeren Krebstherapien kann zu einer geringeren Beeinträchtigung der HRQOL beitragen. Nebenwirkungen sind jedoch nicht gänzlich vermeidbar und müssen gegen die Wirksamkeit der Therapie abgewogen werden. Umso wichtiger für die Verbesserung bzw. den Erhalt der Lebensqualität sind darum supportive Maßnahmen. Ein erster wichtiger Schritt wäre ein Monitoring der Symptome durch systematische Befragung der Patientinnen. In der Praxis zeigt sich jedoch, dass beispielsweise Fatigue zu selten von Ärzten oder Pflegepersonal angesprochen wird. Viele Patientinnen berichten auch nicht auf eigene Initiative darüber [15], teils aus Unwissenheit, dass es sich bei ihren Symptomen um ein potenziell behandelbares Problem handelt, teils aus Mangel an kommunikativen Fähigkeiten oder schlicht aus Mangel an Zeit im Gespräch mit dem Arzt. Als Konsequenz wird dieses stark die Lebensqualität einschränkende Symptom häufig unzureichend behandelt. Eine randomisierte, kontrollierte Studie von Ethan Basch und Kollegen [18] zeigte, dass bereits ein einfaches internetbasiertes Monitoring von 12 häufigen Symptomen, das bei Verschlechterungen eine Meldung an die Study Nurse schickte, bei Patienten im metastasierten Stadium zu signifikanten Verbesserungen der HRQOL und darüber hinaus sogar zu signifikant längerem Überleben führte. Neben dem Symptommonitoring wäre eine Betrachtung von psychosozialen Faktoren sinnvoll, wie frühere oder bestehende depressive
Symptome, Angstzustände, soziale Unterstützung, finanzielle, familiäre oder organisatorische Schwierigkeiten aufgrund der Erkrankung, um bei Bedarf frühzeitig Maßnahmen zum Erhalt der Lebensqualität zu ergreifen, wie beispielsweise den Kontakt zu einer kompetenten Beratungsstelle zu vermitteln.

Darüber hinaus wurden nichtmedikamentöse Interventionen zum Erhalt bzw. zur Verbesserung der HRQOL bzw. zur Reduzierung der Symptomlast in zahlreichen randomisierten, kontrollierten Studien untersucht ( $\triangleright$ Tab. 2). Derzeit liegt hinsichtlich HRQOL gute Evidenz für die Wirksamkeit von Yoga, Kraft-/Ausdauertraining, kognitiver Verhaltenstherapie (CBT) und mindfulness-based stress reduction (MBSR) vor [19-22]. Welche Intervention in der gegebenen Situation am besten wirkt, ist noch unklar. Aktuelle Studien sollen die Interventionen besser auf die jeweiligen individuellen Patienten-, Therapie- und Symptomcharakteristiken abstimmen.

\section{Interessenkonflikt}

Die Autorinnen/Autoren geben an, dass kein Interessenkonflikt besteht.

\section{Literatur}

[1] Kluetz PG et al. Focusing on Core Patient-Reported Outcomes in Cancer Clinical Trials-Response. Clin Cancer Res 2016; 22 (22): 5618

[2] Group W. Study protocol for the World Health Organization project to develop a quality of lifeinstrument (WHOQOL). Quality of Life Research 1993; 2: 153-159

[3] Husson O et al. The EORTC QLQ-C30 Summary Score as Prognostic Factor for Survival of Patients with Cancer in the "Real-World": Results from the Population-Based PROFILES Registry. Oncologist 2019; Epub ahead of print

[4] Waldmann A, Schubert D, Katalinic A. Normative data of the EORTC QLQ-C30 for the German population: a population-based survey. PLoS One 2013; 8 (9): e74149

[5] Mierzynska J et al. Reference values for the EORTC QLQ-C30 in early and metastatic breast cancer. Eur J Cancer 2020; 125: 69-82 
[6] Hamer ] et al. Quality of life (QOL) and symptom burden (SB) in patients with breast cancer. Support Care Cancer 2017; 25 (2): 409-419

[7] Sousa $\mathrm{H}$ et al. A systematic review of factors affecting quality of life after postmastectomy breast reconstruction in women with breast cancer. Psychooncology 2019; 28 (11): 2107-2118

[8] Schmidt ME et al. Determinants of physical, affective, and cognitive fatigue during breast cancer therapy and 12 months follow-up. Int J Cancer 2018; 142 (6): 1148-1157

[9] Koch $L$ et al. Fear of recurrence in long-term breast cancer survivors-still an issue. Results on prevalence, determinants, and the association with quality of life and depression from the cancer survivorship-a multi-regional population-based study. Psychooncology 2014; 23 (5): 547-554

[10] Willson ML et al. Taxanes for adjuvant treatment of early breast cancer. Cochrane Database Syst Rev 2019; 9: CD004421

[11] Chow $S$ et al. Symptoms Predictive of Overall Quality of Life Using the Edmonton Symptom Assessment Scale in Breast Cancer Patients Receiving Radiotherapy. Clin Breast Cancer 2019; 19 (6): 405-410

[12] Schmidt ME, Wiskemann J, Steindorf K. Quality of life, problems, and needs of disease-free breast cancer survivors 5 years after diagnosis. Qual Life Res 2018; 27(8): 2077-2086

[13] Arndt $V$ et al. Quality of life in long-term and very long-term cancer survivors versus population controls in Germany. Acta Oncol 2017; 56 (2): 190-197

[14] Hsu T et al. Quality of life in long-term breast cancer survivors. J Clin Oncol 2013; 31 (28): 3540-3548
[15] de Ligt KM et al. The impact of health symptoms on health-related quality of life in early-stage breast cancer survivors. Breast Cancer Res Treat 2019; 178 (3): 703-711

[16] Bloom JR, Petersen DM, Kang SH. Multi-dimensional quality of life among long-term ( $5+$ years) adult cancer survivors. Psychooncology 2007; 16 (8): 691-706

[17] Davis SR et al. Menopausal symptoms in breast cancer survivors nearly 6 years after diagnosis. Menopause 2014; 21 (10): 1075-1081

[18] Basch E et al. Symptom Monitoring With Patient-Reported Outcomes During Routine Cancer Treatment: A Randomized Controlled Trial. J Clin Oncol 2016; 34 (6): 557-565

[19] Duncan M et al. Review of systematic reviews of non-pharmacological interventions to improve quality of life in cancer survivors. BMJ Open 2017; 7 (11): e015860

[20] Campbell KL et al. Exercise Guidelines for Cancer Survivors: Consensus Statement from International Multidisciplinary Roundtable. Med Sci Sports Exerc 2019; 51 (11): 2375-2390

[21] Danhauer SC et al. Yoga for symptom management in oncology: A review of the evidence base and future directions for research. Cancer 2019; 125 (12): 1979-1989

[22] Matsuda A et al. Effectiveness of psychoeducational support on quality of life in early-stage breast cancer patients: a systematic review and meta-analysis of randomized controlled trials. Qual Life Res 2014; 23 (1): $21-30$ 